\title{
Ultrahigh Frequency Components in the Hot Electron Photomagnetoelectric Response of Strongly Photoexcited Narrow-Gap Semiconductors
}

\author{
E. ShatKovskis ${ }^{a, b, *}$, A. GAlickas ${ }^{b}$ AND O. KipRiJanoviČ ${ }^{b}$ \\ ${ }^{a}$ Vilnius Gediminas Technical University \\ Saulètekio all. 11, Vilnius 2040, Lithuania \\ ${ }^{b}$ Semiconductor Physics Institute, Goštauto 11, Vilnius 01108, Lithuania
}

\begin{abstract}
A photomagnetoelectric effect has been investigated in semiconductors InAs and $\mathrm{Cd}_{x} \mathrm{Hg}_{1-x} \mathrm{Te}(x=0.2$ and 0.26$)$ excited by $Q$-switched neodymium-YAG laser. The photomagnetoelectric signal undergos double-sign-inversion when the intensity of the exciting light pulses exceeds a critical value $I_{\mathrm{c}}=5 \times 10^{24}$ photons $/\left(\mathrm{cm}^{2} \mathrm{~s}\right)$ for InAs and $(1-4) \times 10^{24}$ photons $/\left(\mathrm{cm}^{2} \mathrm{~s}\right)$ for $\mathrm{Cd}_{x} \mathrm{Hg}_{1-x} \mathrm{Te}$ samples. It is shown that a frequency spectrum of photomagnetoelectric response is broadened significantly in the region of high frequencies. In general three frequency bands were distinguished. From this investigation it follows that using laser pulses of a duration $t_{\text {opt }} \sim 1-10 \mathrm{ps}$ the photomagnetoelectric signal in the terahertz range may be generated.
\end{abstract}

PACS numbers: 71.55.Gs, 72.70.Jv, 07.57.Yb, 42.62.Hk

\section{Introduction}

The phenomena resulting from high density of nonequilibrium electron-hole plasma (EHP) and electron heating effects often arise simultaneously when the semiconductor is excited by intense laser light [1-4]. Effects of hot carrier luminescence, hot carrier absorption and complex photomagnetoelectric (PME) effect have been observed in numerous semiconductors as a result [3-5]. In this report, the multiple PME effect has been studied under strong excitation of narrow-gap semiconductors. Nonlinear recombination and heating of dense EHP have been

*corresponding author; e-mail: eusat@fm.vtu.lt; eusat@pfi.lt 
studied in previous papers [5-7], where it was shown that heating of only the electron component of EHP takes place when energy density of the exciting laser pulse was lower than 1-2 MW/ $\mathrm{cm}^{2}$ [4]. The aim of the present paper is to study the time and frequency characteristics of the photoresponse induced by the complex PME effect in narrow-gap semiconductors at intense pulsed laser excitation.

\section{Experiment and analysis procedure}

The specimens of InAs and $\mathrm{Cd}_{x} \mathrm{Hg}_{1-x} \mathrm{Te}(x=0.2$ and 0.26$)$ were placed in the magnetic field of 0.25 T. Either PME electromotive force (emf) or PME current has been measured between ohmic contacts during excitation of samples by $Q$-switched neodymium-YAG laser light pulses of duration 20 to 80 ns. Measurements were done at the room temperature. The evolution of the pulsed photoresponse of the multiple PME signal was observed by broadband registration equipment $[6,7]$. The frequency spectra of the multiple pulse photoresponse was found by a discrete Fourier transform (DFT) procedure. To increase the number of samples and, correspondingly the harmonics number, linear interpolation of discrete function $x[n]$ between nodes $n$ has been introduced. To avoid the Gibbs phenomenon the time windows finitization method was employed [8]: the response function $x[n]$ was multiplied by weight function $w[n]$. The Hamming window was chosen for weighting function, which provides optimum relationship between width of the major lobe and side lobes [9]. The Hamming window function is expressed by formula:

$$
w[n]=0.54+0.46 \cos (2 \pi t[n]) .
$$

Here the designation $t[n]=[n-(N-1) / 2] /(N-1)$ (where $0 \leq k \leq N-1$ and $0 \leq n \leq N-1)$ is used. The procedure allows us to distinguish spectral components $-25 \mathrm{~dB}$ less than the level of major component. Discrete-temporary transform of the consequence weighted by window is expressed in a form of convolution DFT of the signal and DFT of time window. The initiation number of samples was 65 and the frequency of the first harmonic was $25 \mathrm{MHz}$. Evaluation of integrals, which define Fourier coefficients, was realized by numerical integration methods of MathCad media. Spectral density distribution was obtained up to the 40 harmonics or respectively $1000 \mathrm{MHz}$.

\section{Results and discussion}

The amplitude of the complex PME response of two $\mathrm{Cd}_{x} \mathrm{Hg}_{1-x}$ Te crystals as a function of excitation intensity at magnetic field $B=0.25 \mathrm{~T}$ is presented in Fig. 1 . Similar dependences have been observed for InAs crystals. The sign inversion of the response is observable at $I_{\mathrm{c}} \approx(1-4) \times 10^{24}$ photons $/\left(\mathrm{cm}^{2} \mathrm{~s}\right)$ in $\mathrm{Cd}_{x} \mathrm{Hg}_{1-x} \mathrm{Te}$ crystals and at $I_{\mathrm{c}} \approx 5 \times 10^{24}$ photons $/\left(\mathrm{cm}^{2} \mathrm{~s}\right)$ in indium arsenide. The value of $I_{\mathrm{c}}$ 


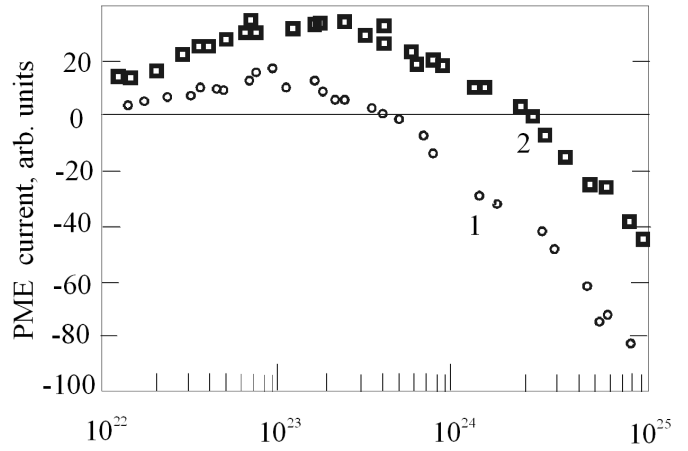

Fig. 1

$\mathrm{I}$, photons $/\left(\mathrm{cm}^{2} \mathrm{~s}\right)$

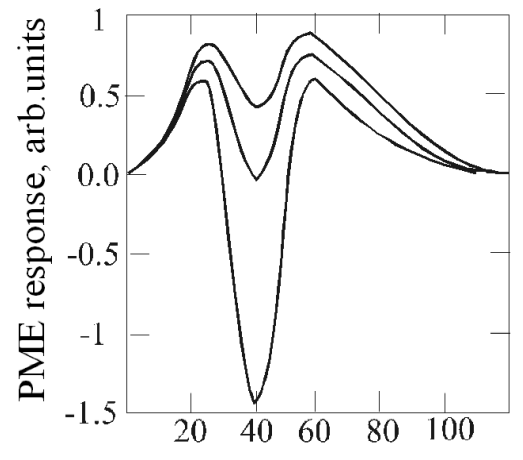

Fig. 2 Time, ns

Fig. 1. Amplitude of multiple PME response current versus intensity of the exciting light in $\mathrm{Cd}_{x} \mathrm{Hg}_{1-x}$ Te crystals: $1-x=0.2 ; 2-x=0.26$.

Fig. 2. The shape of the optically induced PME signal in the vicinity of $I_{\mathrm{c}}$ shown for $I<I_{\mathrm{c}}, I=I_{\mathrm{c}}$ and $I>I_{\mathrm{c}}$.

slightly increases if a magnetic field is increased. This property is easily explained if one takes into account different dependences of PME components on magnetic field [7]. Figure 2 demonstrates time-dependence of PME response in the vicinity of $I_{\mathrm{c}}$. Such shape of the response can be understood if one refers to Fig. 1 and remembers that the laser pulse is bell-like. Due to the strong dependence of EHP temperature on the excitation intensity, the sharpening of the response signal occurs. Consequently the PME response may involve more frequent harmonics than those in the harmonic spectra of the envelope line of the optical pulse.
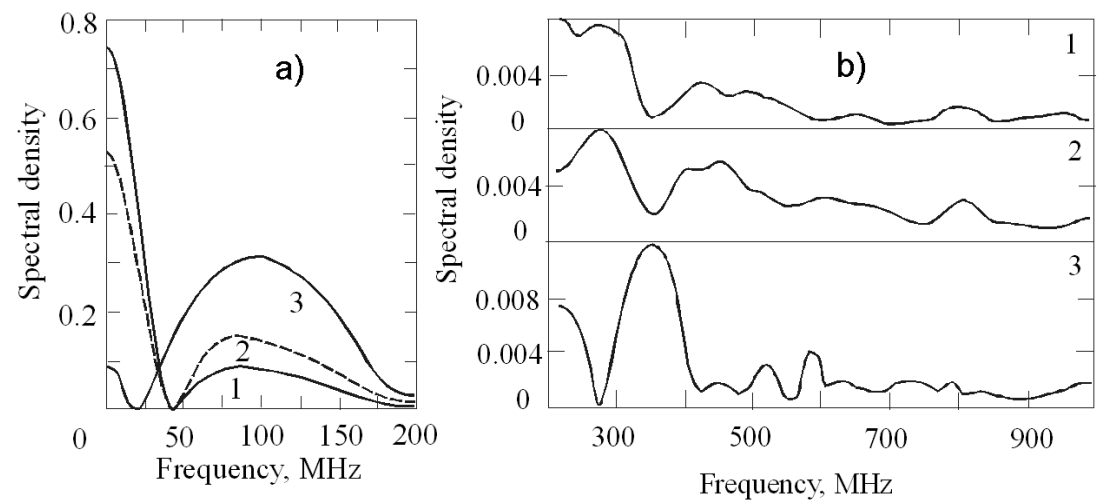

Fig. 3. (a) Main part of PME spectrum; (b) High frequency components of PME spectrum. $1-I<I_{\mathrm{c}} ; 2-I=I_{\mathrm{c}} ; 3-I>I_{\mathrm{c}}$.

The DFT spectra of PME signals are shown in Fig. 3a, b. The main part of the spectral density corresponds to the envelope line of the optical pulse. The rise in the exciting light intensity causes an increase of negative part in PME 
signals. It lowers the low frequency component amplitudes in spectral density and governs the growth of higher frequency ones (see Fig. 3a). Spectral density of the high frequency region is shown in Fig. 3b. The frequency spectra is broadened significantly in the region of high frequencies because of the pulse sharpening effect as well as of the fact that the PME response involves both positive and negative parts. The central frequency $\nu_{\mathrm{hf}}$ of the most high frequency band can be expressed as $\nu_{\mathrm{hf}} \approx 10 / t_{\mathrm{opt}}$ (here $t_{\mathrm{opt}}$ is the duration of laser pulse) in the case of $I>I_{\mathrm{c}}$. Hence, using the laser pulses of duration $t_{\text {opt }} \sim 1-10 \mathrm{ps}$, the terahertz frequency range may be achieved. Because of rather uniform spectral density in a wide frequency range the case $I=I_{\mathrm{c}}$ looks to be attractive too.

\section{Conclusion}

The obtained results show that time response of the PME signal inverts its sign two times if a narrow-gap semiconductor is strongly excited. The frequency spectra of such signals are wider. This property allows us to widen the frequency range that is governed by the envelope of the exciting optical pulse.

\section{References}

[1] T.M. Rice, J.C. Hensel, T.G. Philips, S.A. Thomas, The Electron-Hole Liquid in Semiconductors, Acad. Press, New York 1977.

[2] G.N. Galkin, Trudy FIAN 28, 3 (1981) (in Russian).

[3] J. Shah, Solid St. Electron. 32, 1051 (1989).

[4] E. Shatkovskis, Mater. Sci. Forum 297-298, 11 (1999).

[5] S.A. Lyon, J. Lumin. 35, 121 (1986).

[6] E. Shatkovskii, A. Tamaševičius, Phys. Status Solidi A 42, 111 (1977).

[7] E. Shatkovskii, A. Tamaševičius, E. Matulionis, Phys. Status Solidi A 51, K97 (1979).

[8] L. Rabiner, B. Gould, Theory and Application of Digital Signal Processing, Prentice-Hall, Inc., Engelwood Cliffs (New Jersey) 1975.

[9] R.J. Higgins, Amer. J. Phys. 44, 766 (1976). 